\title{
Lossless Holographic Spectrum Splitter In Lateral Photovoltaic Devices
}

Di Lin, Ethan R. Torrey, James R. Leger, and Phil. I. Cohen

Department of Electrical and Computer Engineering, University of Minnesota, Minneapolis, MN 55455

\section{Background}

Lateral photovoltaic (PV) structures require efficient partitioning of the solar spectrum and light delivery to the respective cells. Single-junction cells can be used, eliminating the need for current and lattice matching. We investigate the design issues associated with multiplexed volume holograms in fulfilling this role in a two-cell lateral PV system.

\section{Designing Holographic Spectrum Splitters}

- Phase volume gratings can be used to redirect a segment of the incident spectrum without loss. The diffraction coefficient of a single volume grating can be calculated using Kogelnik's coupled wave analysis [1].

- The spectral diffraction characteristics of the hologram can be engineered by multiplexing several volume phase gratings in the same hologram.

- Kogelnik's equations do not account for coupling effects that occur in multiplexed volume gratings. We have generalized this theory to include multiplexed gratings and calculate the resulting diffraction characteristics.

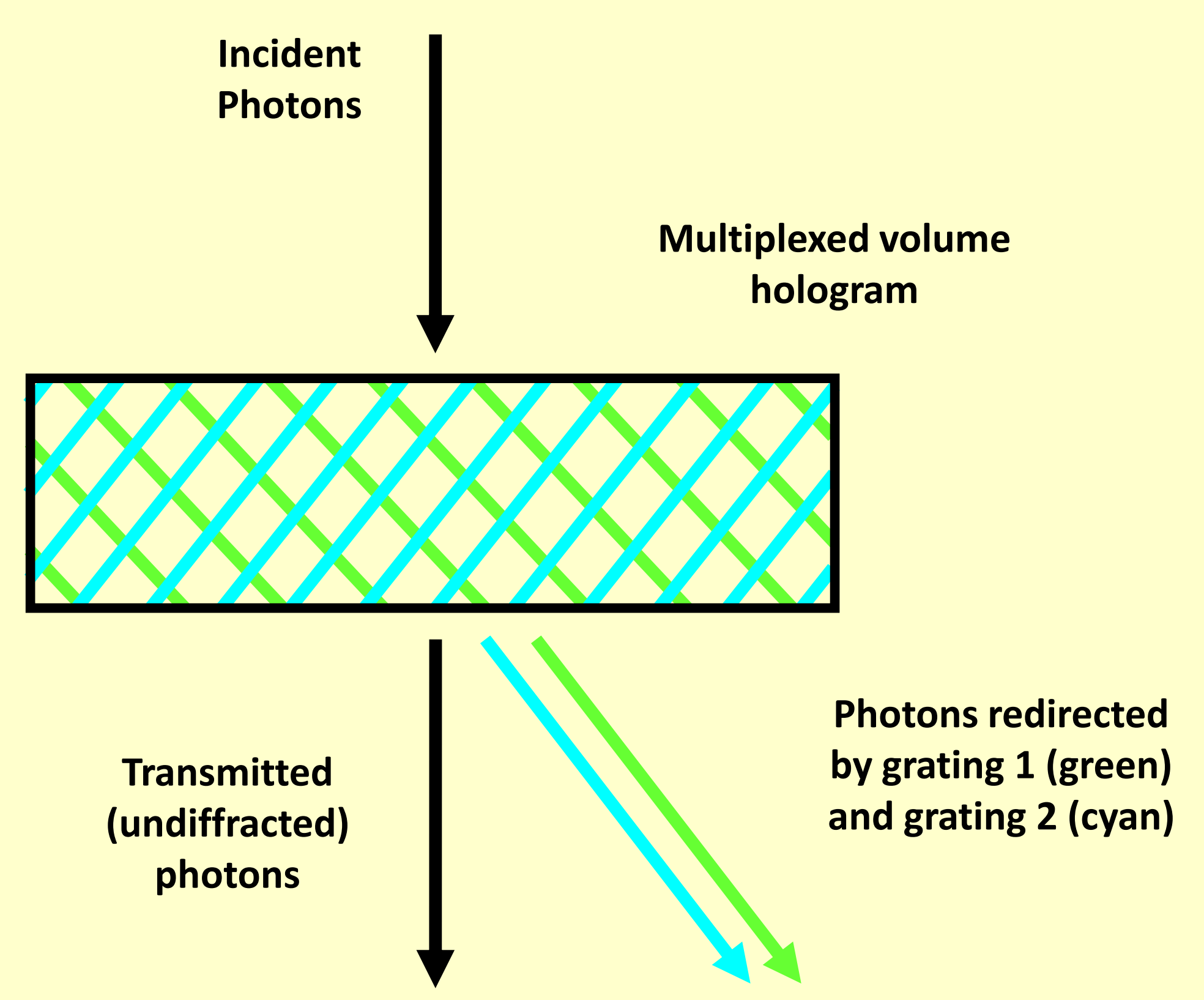

Figure 2. Diffraction geometry of two multiplexed volume gratings

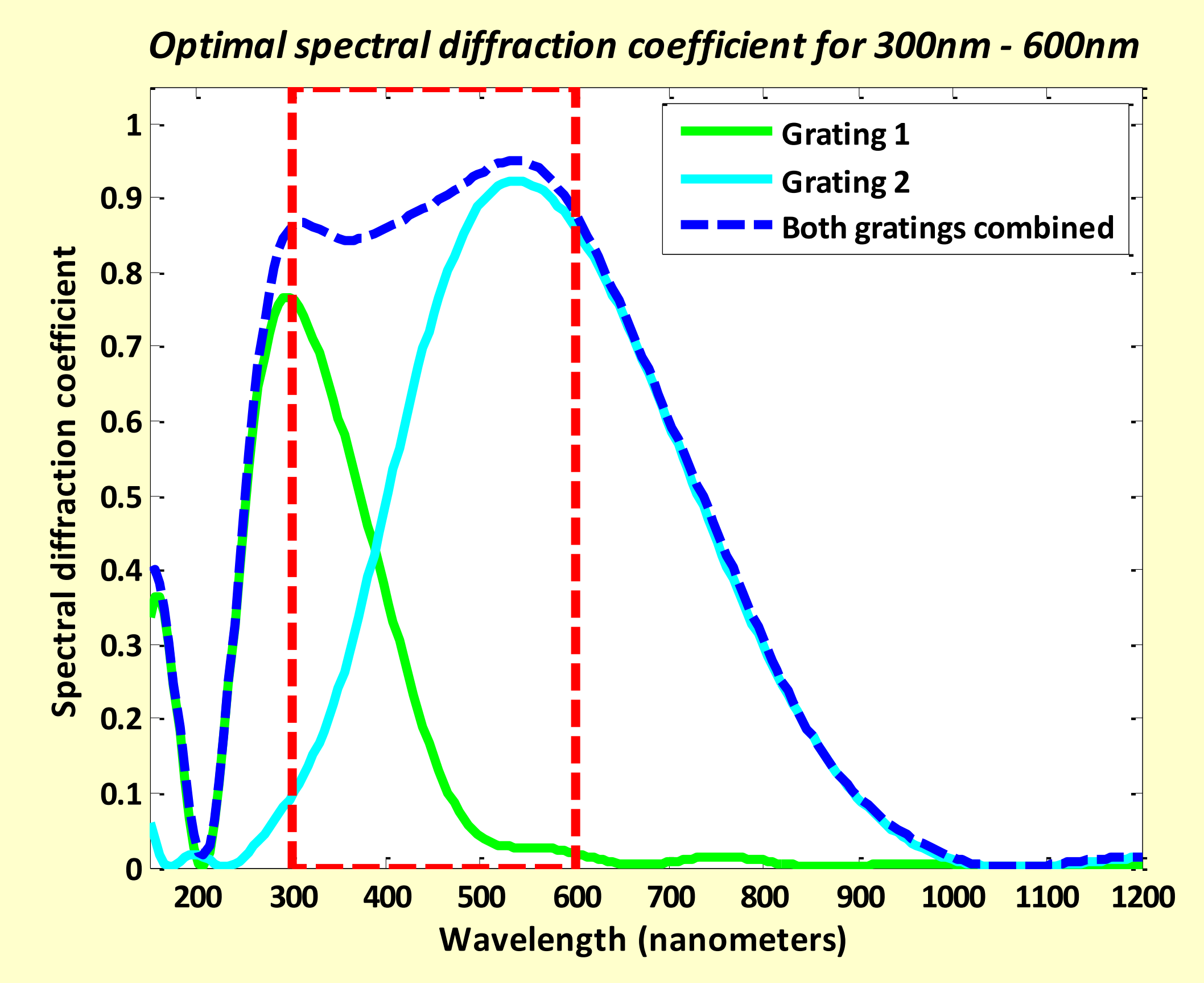

Figure 4. Center wavelengths and spectral bandwidths of two multiplexed volume gratings are optimized to maximize the overall diffraction coefficient between $300 \mathrm{~nm}$ and $600 \mathrm{~nm}$.

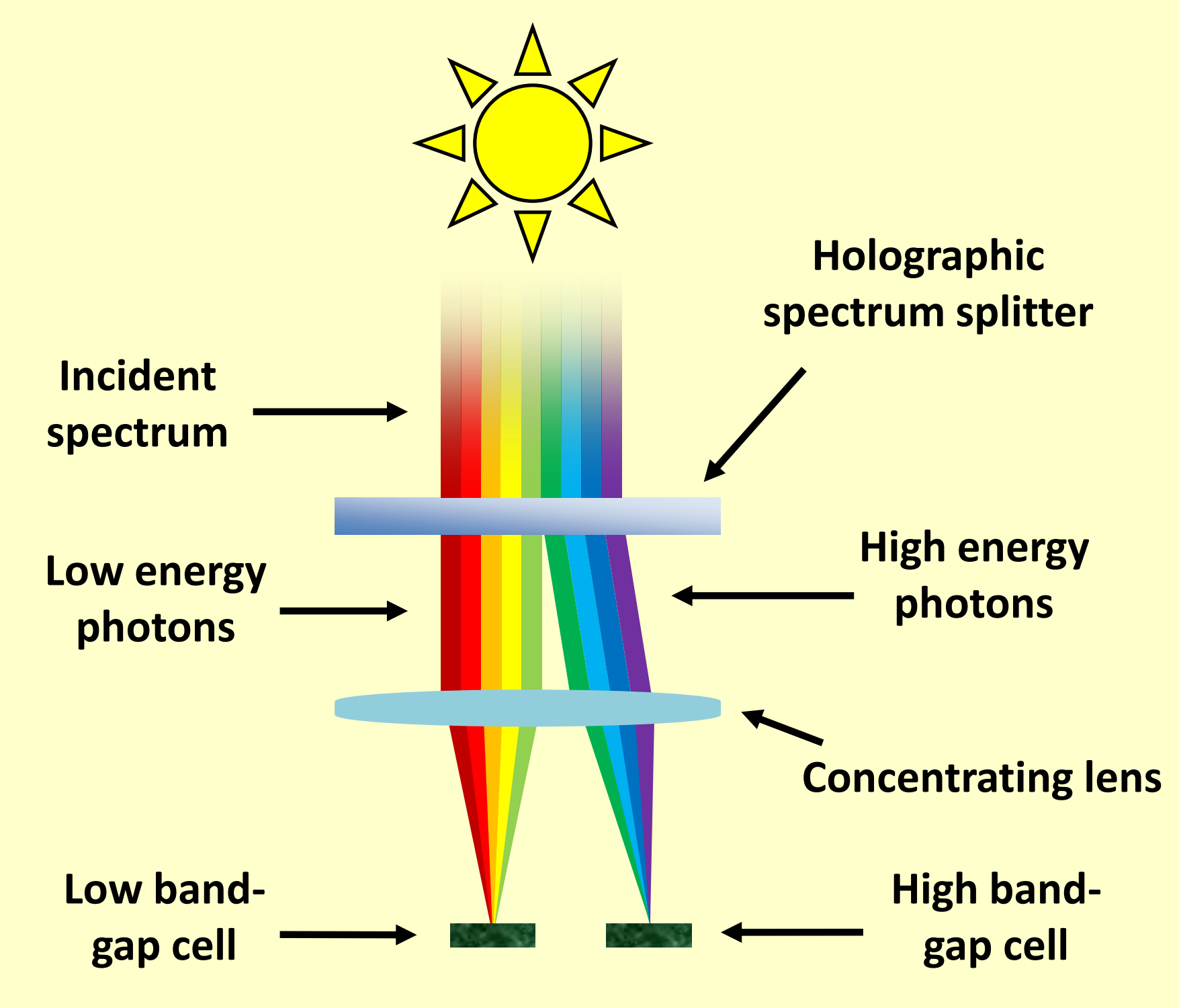

Figure 1. Schematic of the proposed two-cell lateral PV device
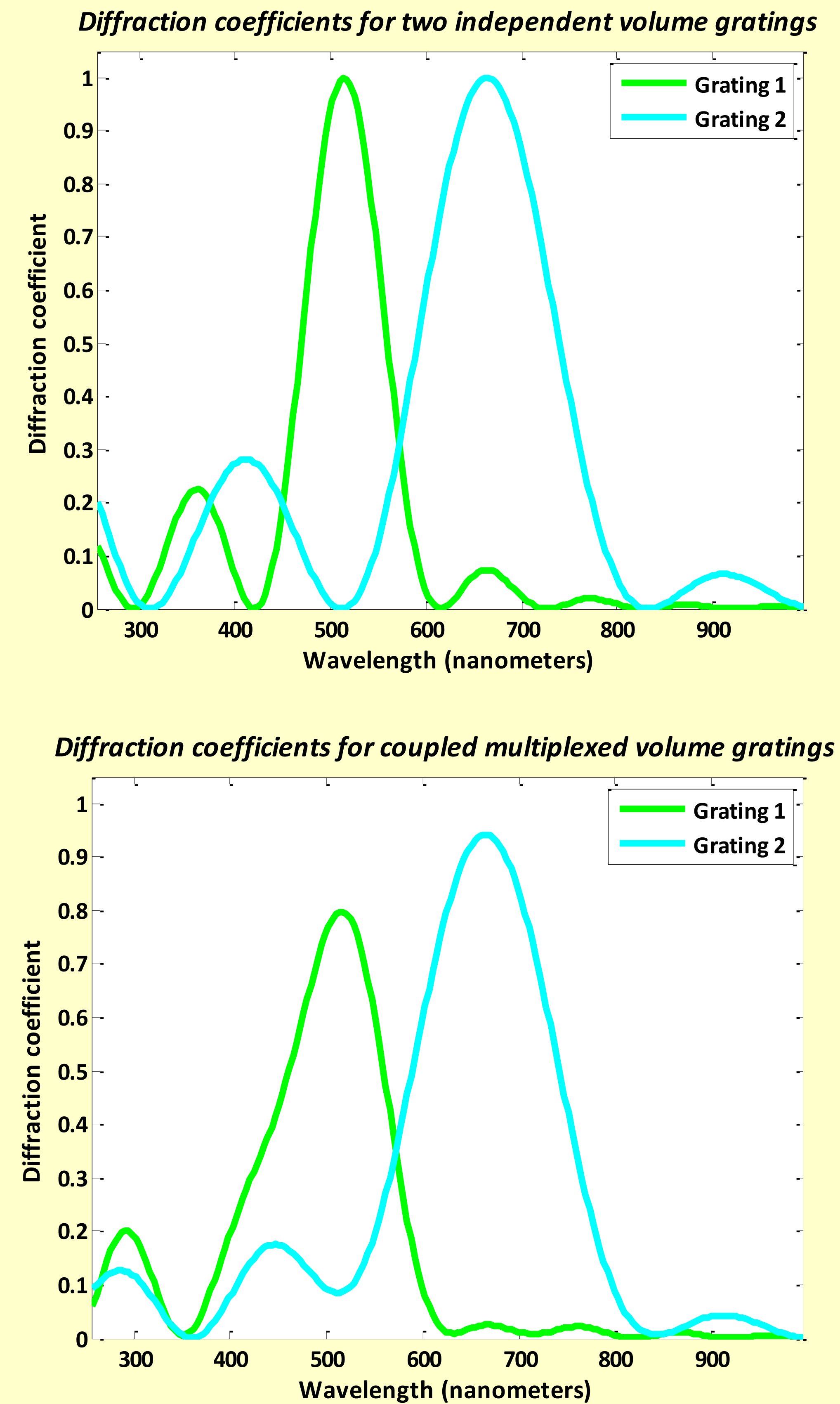

Figure 3. Comparison between the spectral diffraction characteristics calculated using Kogelnik's equations (top) and our coupled grating model (bottom).

Using our coupled grating model, we can maximize the overall spectral diffraction coefficient within a defined spectral band.

- Nearly $90 \%$ of the incident power within a $300 \mathrm{~nm}$ spectral bandwidth can be redirected to the high band-gap cell using only two multiplexed volume gratings (Figure 4).

The remaining $10 \%$ is transmitted through the hologram and is collected by the low band-gap cell.

\section{Detailed Balance Optimization}

Redirected photons with energies below the band-gap of the low band-gap cell cannot be absorbed.

- This means a gradual fall-off in the hologram's spectral diffraction coefficient (as photon energy decreases) is detrimental to device efficiency.

- The spectral diffraction characteristics of the hologram must be optimized concurrently with band-gap selection by using a detailed balance calculation.

- The optimal partitioned spectral segments directed at each of the single-junction lateral cells are shown in Figure 6.

- Band-gap energies for the lateral cells are optimized at $0.94 \mathrm{eV}$ and $1.77 \mathrm{eV}$.

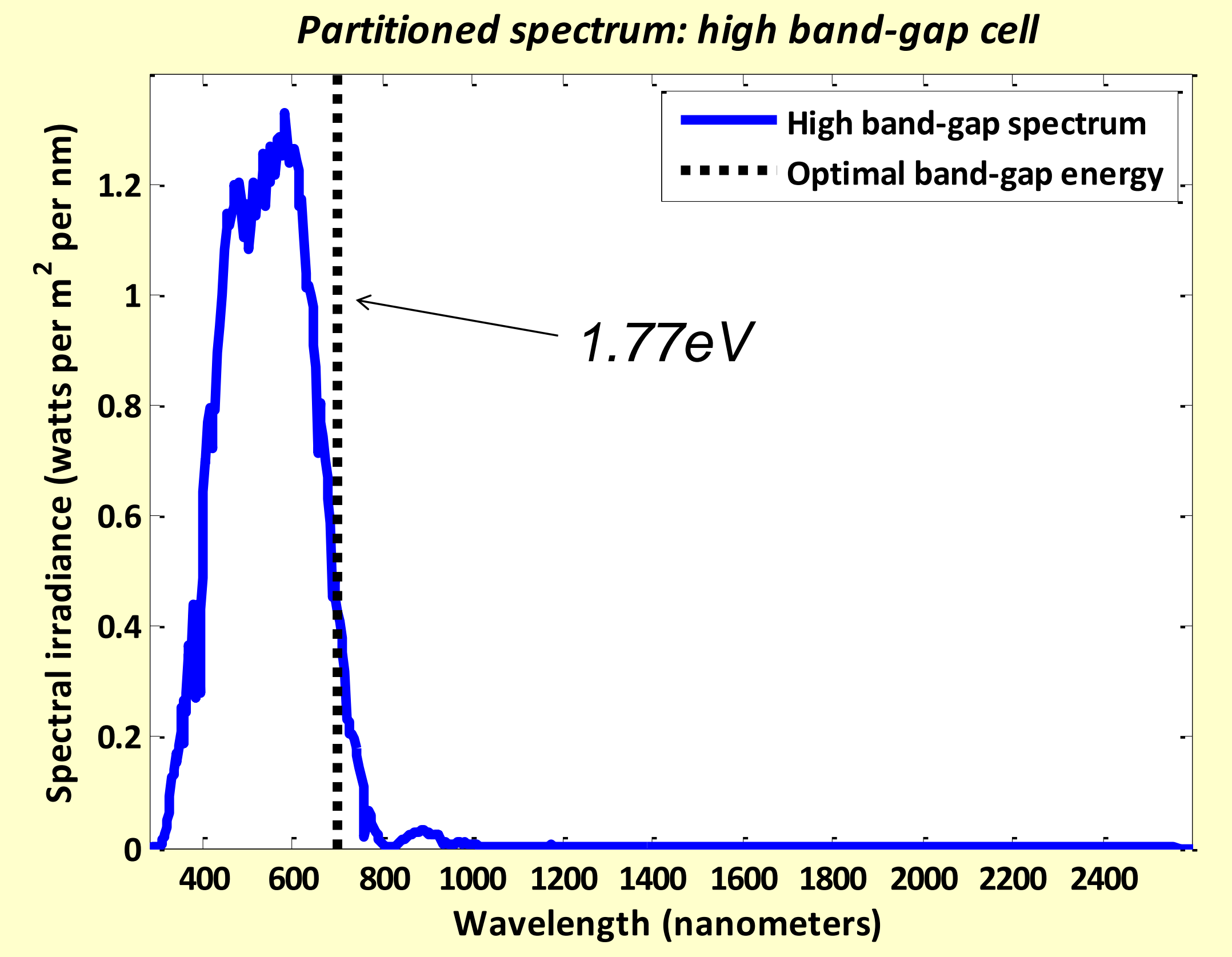

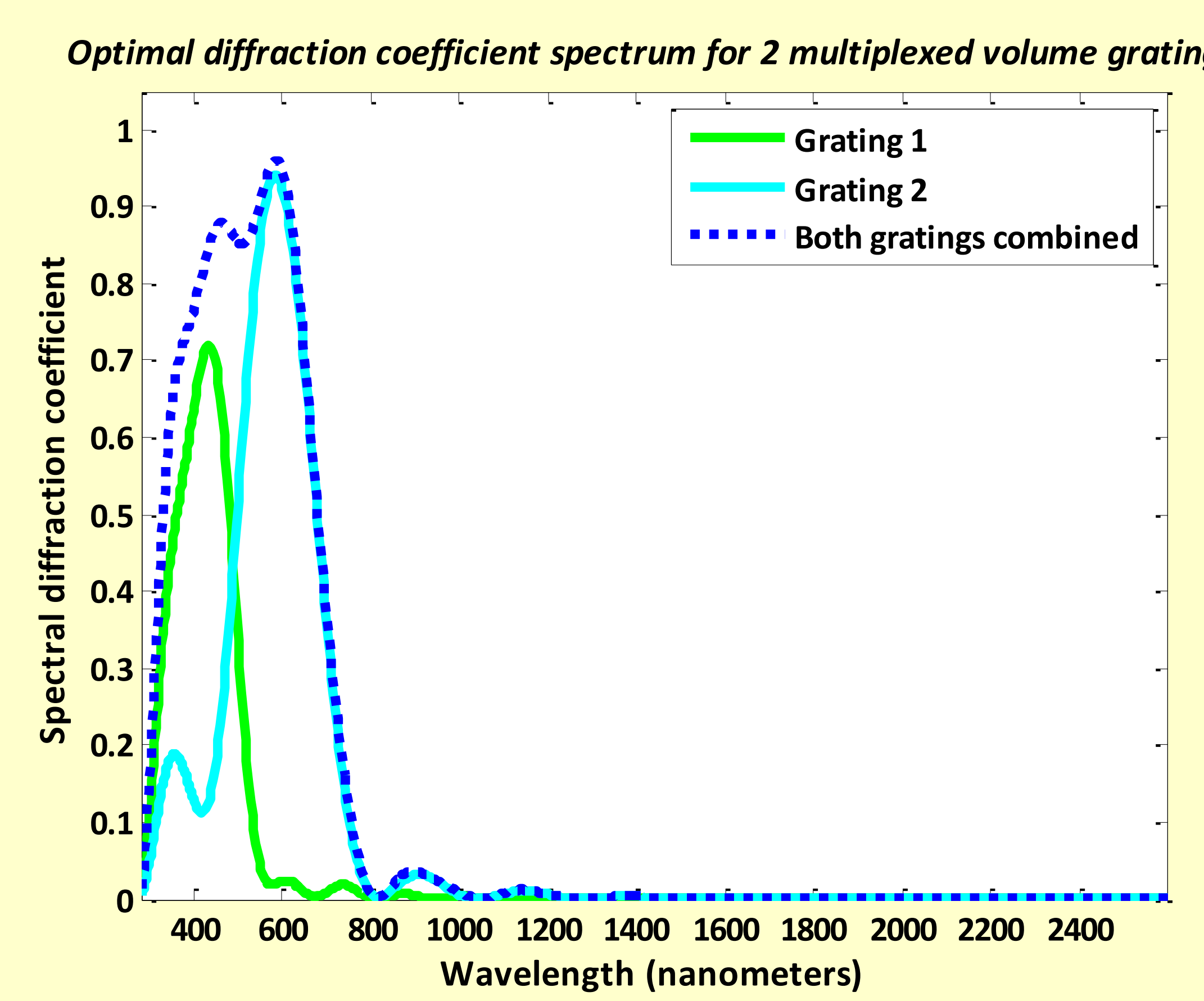

Figure 5. Spectral characteristics of the hologram consisting of two multiplexed volume gratings, aimed at maximizing device efficiency.

Figure 6. Partitioned solar spectrum directed to the high band-gap cell (left) and the low band-gap cell (right) for the AM .15 direct-plus-circumsolar spectrum.

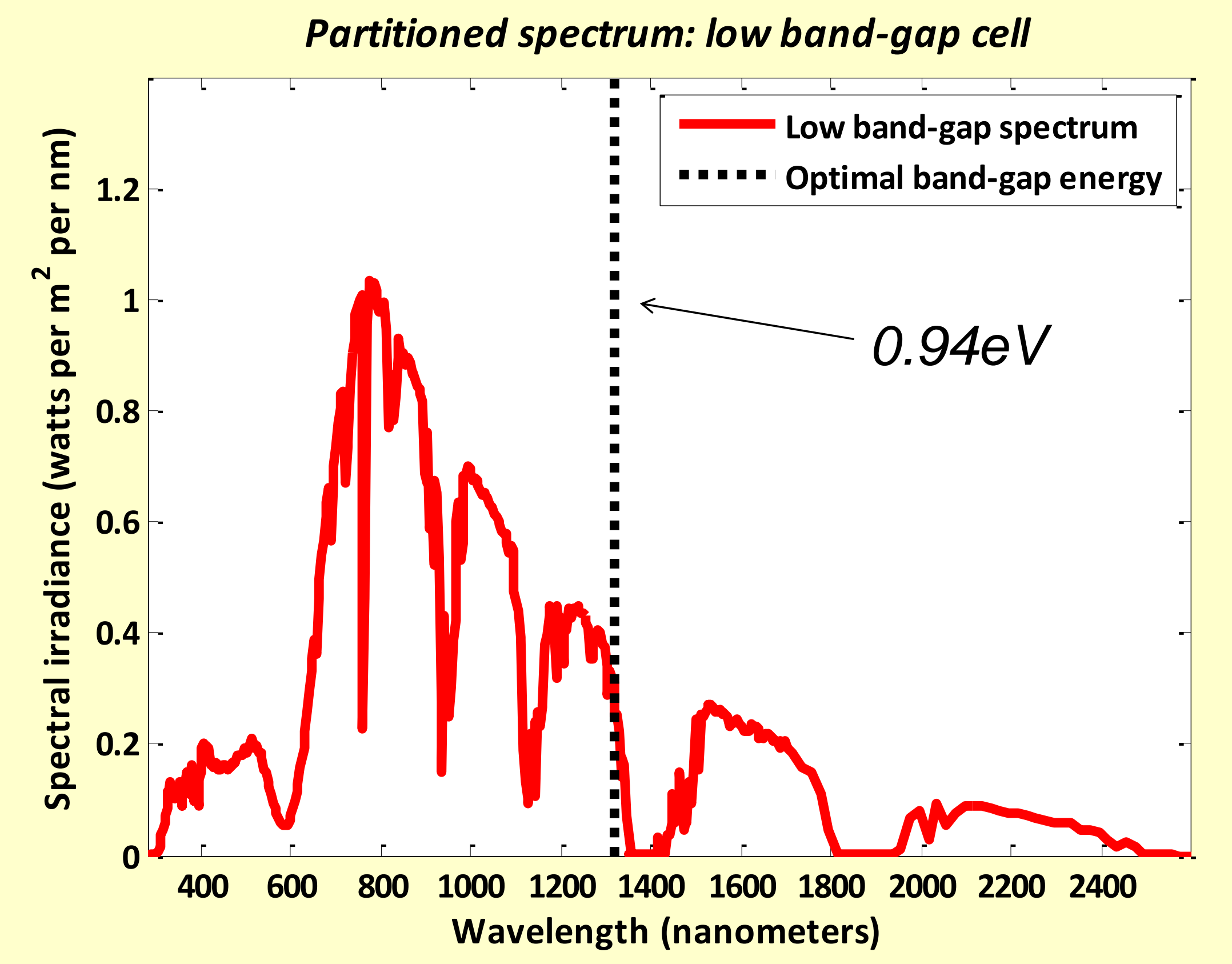

\section{Device Performance}

The theoretical maximum efficiency for the proposed two-cell lateral device is $45.0 \%$ under the following assumptions:

- incident AM 1.5 direct-plus-circumsolar spectrum

- concentration of 100 suns (at each wavelength)

- lateral cells illuminated from one side only

photons emitted due to radiative recombination are not recycled

Performance is comparable to the theoretical maximum achieved using tandem structures, especially when diurnal and seasonal variations are considered. However, the lateral architecture offers a significant simplification in PV fabrication. Tandems with a series current constraint typically achieve $43 \%$ - $45 \%$ under the same assumptions [2].

\section{References}

[1] H. Kogelnik, "Coupled Wave Theory for Thick Hologram Gratings", Bell System Tech. J. 48, 2909-2947 (1969).

[2] E.R. Torrey, P.P. Ruden, P.I. Cohen, "Performance of a split-spectrum photovoltaic device under timevarying spectral conditions", J. Appl. Phys. 109, 074909 (2011) 